\title{
Control of interlinking converters in hybrid AC/DC grids: network stability and scalability
}

\author{
Jeremy D. Watson and Ioannis Lestas
}

\begin{abstract}
Hybrid AC/DC networks are an effective solution for future power systems, due to their ability to combine advantages of both AC and DC networks. However, they bring new technological challenges, one key area being the control of such a network. The network, and especially the interlinking converter (ILC), must be controlled to ensure that the DC and AC subsystems coordinate to stabilize the network and allocate power appropriately. This is an area which has attracted considerable recent interest due to the non-triviality of the control design. One promising tool is passivity theory which allows the derivation of decentralized conditions through which the stability of the network can be guaranteed. This paper investigates the application of a passivity framework to AC/DC grids, using a typical lossless line assumption. By ensuring that an appropriately formulated passivity condition is satisfied by the $\mathrm{AC}$ and DC buses, and the interlinking converter, the stability of the interconnection can be guaranteed. We also discuss how the ILC controller may be designed to achieve an appropriate power allocation between $\mathrm{AC}$ and DC sources. Simulation results demonstrate that the proposed ILC control design regulates the frequency and voltages of the hybrid AC/DC network with a stable operation maintained.
\end{abstract}

\section{INTRODUCTION}

Hybrid AC/DC grids are rapidly gaining attention as an effective solution for future power systems, due to their ability to combine advantages of both $\mathrm{AC}$ and $\mathrm{DC}$ networks. AC technology is well established, whereas DC networks have lower losses (mainly due to the absence of reactive power), and higher power transfer capability [1]-[2]. In addition, DC networks can facilitate the connection of energy sources such as solar and wind, and certain large loads. By using highefficiency converters to connect the $\mathrm{AC}$ and $\mathrm{DC}$ grids, the overall efficiency of the network can be improved. HVDC transmission is already a key technology for flexible and controllable power transfer, and it is envisaged that future transmission systems will consist of $\mathrm{AC}$ and DC subgrids connected to form hybrid AC/DC grids [3]. Hybrid AC/DC networks bring new technological challenges, of which one key area is the control of such a network [4]. The network, and in particular the interlinking converter (ILC), must be controlled in such a way as to ensure that the DC and AC subsystems coordinate to stabilize the network and allocate power appropriately across the hybrid power grid [5]. This is a non-trivial problem which is made more difficult by the fact that DC networks typically have faster response times compared to $\mathrm{AC}$ networks, and the interactions between the DC and AC sides can be destabilizing [6],[7].

Jeremy D. Watson and Ioannis Lestas are with the Department of Engineering, University of Cambridge, Trumpington Street, Cambridge, CB2 1PZ, United Kingdom. This work was supported by ERC starting grant 679774 .
The control of the interlinking converter is therefore a key issue for hybrid AC/DC approaches. Several control approaches have been proposed, including traditional dualdroop control (e.g. [9]), integral dual-droop control [10][13], and approaches based on matching control [14]. While the standard dual-droop approach is logical, the two droop schemes tend to interfere with each other and degrade powersharing performance [9]. In our former paper [14], we focused on achieving suitable power sharing for both primary and secondary control with a simple converter model. However, guaranteeing stability of the network becomes an involved problem when the ILC dynamics are taken into account, and potential stability issues have been reported in hybrid AC/DC networks, e.g. [15], [16], [17]. The timescale difference between slower AC responses and faster DC responses can be a challenge for the control of hybrid AC/DC grids, and inappropriate control design can lead to poor transient performance and oscillations. Hence systematic analysis and design approaches need to be developed.

One potential and promising tool to ensure stability in a scalable way is passivity theory. Passivity-based approaches have been proposed when AC networks (e.g. [20], [21], [22], [23], [24]) or DC networks (e.g. [25], [26], [27]) are individually studied. The stability of grid-connected converters may be analyzed via various other methods when a centralized analysis is carried out, e.g. by directly modelling the converters and grid, or by using the impedance-based multivariable Nyquist criterion in [28]. However a distinctive feature of a passivity approach is the fact that it leads to decentralized stability conditions that involve only local bus dynamics. This is an important property when large scale networks are analyzed involving potentially multiple converters and energy sources, as it can ensure system stability when there are grid variations or converters are added/removed, by satisfying decentralized conditions on the bus dynamics. On the other hand, passivity is only a sufficient condition for stability (as is the case with any decentralized stability condition), therefore appropriate control designs need to be developed such that the conditions it leads to are satisfied.

This paper will investigate the application of a passivity framework to AC/DC grids. This is a problem that has not been studied thus far in the literature, with decentralized passivity based designs having been applied to cases where AC or DC networks are individually studied. In a hybrid AC/DC network the interlinking converter (ILC) must satisfy appropriate passivity properties on the way its dynamics relate the current and voltages in the $\mathrm{AC}$ and $\mathrm{DC}$ sides of the converter respectively. This is in general a challenging problem 
and in this paper we investigate the feasibility of such a passivity-based approach for controller design. By ensuring an appropriately formulated passivity criterion is satisfied by the AC network (with an assumption of lossless transmission lines), the DC network, and by the interlinking converter, the stability of the interconnection can be guaranteed. Another important factor for the control of the ILCs is the powersharing properties within the network. Traditional dual-droop approaches are unable to share power across the entire hybrid AC/DC network effectively [5],[14], and this can result in overloading of local sources. This paper will consider various control designs to achieve good power-sharing performance while satisfying a decentralized passivity based stability condition, which is a problem that has not been addressed thus far in the literature.

To summarize, the contributions of this paper are:

1) The application of passivity theory to hybrid AC/DC grids. Focusing especially on the ILC, we find a decentralized condition by which stability may be guaranteed.

2) We consider the design of ILC controllers in order to satisfy this condition while achieving appropriate power-sharing properties. Our analysis reveals a tradeoff between network stability and power-sharing.

3) We propose an ILC controller to achieve our control objectives and illustrate the effectiveness of our approach with numerical simulations.

The paper is organized as follows. The background is given in Sections II-IV. In particular, Section II presents the notation used in the paper, section III provides an overview of passivity approaches and how they can be extended to hybrid AC/DC networks, and section IV presents the mathematical models used for the network. The problem is posed in section $\mathrm{V}$, where a main stability result is presented and a discussion is included on various ILC control schemes. Simulations in section VI illustrate the applicability of our proposed design and our conclusions are given in section VII.

\section{PRELIMINARIES AND NOTATION}

We consider a general hybrid AC/DC network with the set of buses denoted by $N=(1,2, \ldots,|N|)$ and the set of transmission lines by $E=(1,2, \ldots,|E|)$. For each bus $j \in N$ we use $i: i \rightarrow j$ and $k: j \rightarrow k$ to denote the predecessors and successors of bus $j$ respectively ${ }^{1}$. We also define the set of all AC buses $N_{a c}$ and all DC buses $N_{d c}$, such that $N_{a c} \cup N_{d c}=N$; likewise we define the set of all AC edges $E_{a c}$ and all DC edges $E_{d c}$. The network is composed of multiple AC and DC subsystems and connections between AC and DC buses are facilitated by the interlinking converters, the set of which is denoted by $X=(1,2, \ldots,|X|)$. The ILC buses are denoted by $X_{j}^{a c} \in N_{a c}$ and $X_{j}^{d c} \in N_{d c}$ for the AC and DC buses, respectively, to which the ILC $j$ is connected. The set of $\mathrm{AC}$ buses to which a converter is connected is denoted by $X_{a c}=\left(X_{1}^{a c}, \ldots, X_{|X|}^{a c}\right) \subset N_{a c}$. Similarly, the set of DC buses to which a converter is connected is denoted by $X_{d c}=\left(X_{1}^{d c}, \ldots, X_{|X|}^{d c}\right) \subset N_{d c}$. For convenience, we

${ }^{1}$ The results presented in the paper are unaffected by the choice of direction. define the set of DC buses excluding the converter buses by $\mathbb{G}_{d c}=N_{d c} \backslash X_{d c}$.

Table I summarizes additional notation that will be used throughout the paper. In particular, these are parameters associated with the system model that will be described in section IV. Note that the variables (i.e. $\omega_{j}, \eta_{i j}, V_{j}^{a c}, p_{j}^{G}, p_{j}^{L}$, $p_{j}^{X}, p_{j}, q_{j}^{G}, q_{j}^{L}, q_{j}^{X}, q_{j}, p_{i j}, q_{i j}, i_{i j}$ and $\left.V_{j}^{d c}\right)$ in Table I refer to deviations from a corresponding nominal ${ }^{2}$ value.

Table I

NOTATION IN SYSTEM MODEL.

\begin{tabular}{cl}
\hline$\omega_{j}$ & AC frequency at bus $j \in N_{a c}$ \\
$\eta_{i j}$ & voltage angle difference between two AC buses $i$ and $j$ \\
$V_{j}^{a c}$ & AC voltage at bus $j \in N_{a c}$ \\
$M_{j}$ & inertia at bus $j \in \mathbb{N}_{a c}$ \\
$D_{j}$ & damping coefficient at bus $j \in \mathbb{N}_{a c}$ \\
$\tau_{j}^{g}$ & generator time constant at bus $j \in \mathbb{N}_{a c}$ \\
$a_{j}, b_{j}$ & constants in the third-order generator model \\
$\tau_{j}^{x}$ & interlinking converter time constant at bus $j \in X_{a c}$ \\
$n_{i}$ & reactive power - voltage droop coefficient for the ILC \\
$X_{j}$ & generator d-axis reactance constant \\
$p_{j}^{G}$ & generated active power at bus $j$ \\
$p_{j}^{L}$ & load active power at bus $j$ \\
$p_{j}^{X}$ & interlinking converter active power transfer (DC to AC) at bus $j$ \\
$p_{j}$ & total active power injected into the network at bus $j \in N_{a c}$ \\
$q_{j}^{G}$ & reactive power injected into the network at bus $j \in N_{a c}$ \\
$q_{j}^{L}$ & reactive power absorbed at bus $j \in N_{a c}$ \\
$q_{j}^{X}$ & reactive power injected by interlinking converter at bus $j \in N_{a c}$ \\
$q_{j}$ & total reactive power injected into the network at bus $j \in N_{a c}$ \\
$p_{i j}$ & active power transfer between buses $i$ and $j$ for $(i, j) \in E_{a c}$ \\
$q_{i j}$ & reactive power transfer between buses $i$ and $j$ for $(i, j) \in E_{a c}$ \\
$B_{i j}$ & transmission line susceptance for $(i, j) \in E_{a c}$ \\
$C_{j}$ & capacitance at bus $j \in N_{d c}$ \\
$V_{j}^{d c}$ & DC voltage at bus $j \in N_{d c}$ \\
$i_{i j}$ & DC current between bus $i$ and $j$ for $(i, j) \in E_{d c}$ \\
$G_{i j}$ & line conductance for $(i, j) \in E_{d c}$ \\
\hline &
\end{tabular}

\section{PASSIVITY CONDITIONS FOR HYBRID AC/DC NETWORKS}

In this section we provide an overview of how passivity approaches have been successfully employed in the literature for decentralized control design in DC and AC networks. We then discuss how these ideas can be extended in a hybrid AC/DC network, whereby an appropriate passivity condition on the ILC can be formulated. The network model we will consider is described in detail in section IV where a stability result will be stated, with a corresponding proof provided.

\section{A. Passivity}

Passivity approaches have been widely used in the literature to achieve network stability in a decentralized and scalable

\footnotetext{
${ }^{2}$ A nominal value of a variable is defined as its value at a suitable equilibrium of the system with $\mathrm{AC}$ frequency equal to the nominal value $(50 \mathrm{~Hz}$ or $60 \mathrm{~Hz})$. In practice, these nominal values may also be related to the control design, e.g. power, voltage or frequency reference values for droop control.

${ }^{3}$ To be precise, this is the difference between the synchronous and transient reactances.
} 
way. The key property is the fact that the negative feedback interconnection of two passive systems is stable and passive [29]. Therefore, by representing the system dynamics as an interconnection of two subsystems and ensuring that both are passive with respect to their inputs and outputs, we can guarantee stability. A main feature in power systems that can be exploited is the fact that transmission lines dynamics have natural passivity properties. Hence if the network is viewed as the interconnection of two multivariable systems, one associated with the transmission lines and one associated with the bus dynamics then one can deduce that passivation of the bus dynamics is sufficient for stability ${ }^{4}$. In the following sections we will review such conditions for DC and AC networks and discuss how these can be extended for hybrid AC/DC networks.

We provide first a definition of what is meant by a passive system. Consider a dynamical system with $m$ inputs, $n$ states, and $m$ outputs of the form:

$$
\begin{aligned}
\dot{x} & =f(x, u) \\
y & =g(x, u)
\end{aligned}
$$

where $u(t) \in \mathbb{R}^{m}$ is the input vector, $x(t) \in \mathbb{R}^{n}$ the vector of states, and $y(t) \in \mathbb{R}^{m}$ the output, $f$ is locally Lipschitz, and $g$ is continuous.

Definition 1 (Passivity): The system (1) is locally passive about an equilibrium point with constant input $\bar{u}$ and constant state $\bar{x}$ if there exists open neighbourhoods $U$ of $\bar{u}$ and $X$ of $\bar{x}$ and a continuously differentiable function $W(x)$ such that

$$
\dot{W}(x) \leq(u-\bar{u})^{T}(y-\bar{y}) .
$$

for all $x \in X$ and $u \in U$ The system is locally input strictly passive if the definition above holds with the right hand side of (2) replaced by $(u-\bar{u})^{T}(y-\bar{y})-\phi(u-\bar{u})$ where $\phi$ is a positive definite function.

Remark 1: It should be noted that if system (1) is linear then if the passivity property holds locally about an equilibrium point, it also holds globally, i.e., for any other equilibrium point and for any deviation from an equilibrium point. Therefore for linear systems which satisfy Definition 1, we will refer to them as being "passive" (or "input strictly passive") without making reference to a particular equilibrium point.

Remark 2: It should be noted that for linear systems $W(x)$ can be chosen to be a quadratic function with a strict minimum at the equilibrium point, when the system is controllable and observable. In the rest of the paper we will assume that $W(x)$ has this property when Definition 1 is satisfied by a linear system.

Remark 3: The passivity property may easily be verified for linear systems via the KYP Lemma that yields a linear matrix inequality (LMI) feasibility problem. Note also that for linear systems passivity is equivalent to the positive realness of the corresponding transfer matrix [29].

In this paper we will show that an appropriately formulated passivity property on the ILC can be used to deduce stability

\footnotetext{
${ }^{4}$ Note that this interpretation is also closely linked to Lyapunov arguments as the summation of the storage functions of the passive systems is a candidate Lyapunov function.
}

of the hybrid AC/DC network. Furthermore, we will discuss how existing ILC control schemes can be adjusted to satisfy the decentralized condition relating to the passivity property.

\section{B. DC network passivity}

Passivity approaches for DC networks typically use the current and voltages as inputs and outputs [25], [30], as illustrated in Fig. 1. The resulting transfer function is therefore often called the "impedance" or "admittance", since it relates voltages and currents. In particular, the transmission line dynamics are passive when viewed as a dynamical system with input the vector of bus voltage deviations $V^{d c}=\left\{V_{1}^{d c}, V_{2}^{d c}, \ldots\right\}$ and output the vector of bus injection current deviations $i^{d c}=\left\{i_{1}^{d c}, i_{2}^{d c}, \ldots\right\}$ (follows easily from the fact that these are composed only of RLC components).

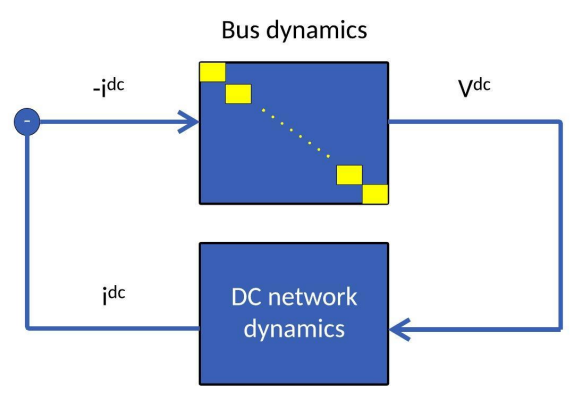

Figure 1. DC grid representation as feedback interconnection of Network/Bus subsystems.

The multivariate system representing aggregate bus dynamics has a diagonal structure. Hence stability of an equilibrium point of the interconnection can be deduced if for each bus $j$ the dynamical system with input $-i_{j}^{d c}$ and output $V_{j}^{d c}$ is strictly passive about this point.

\section{AC network passivity}

Passivity-based approaches in AC networks have been proposed in the literature, e.g. [21], [23], [24] to design converter and generator control mechanisms. Unlike DC networks, the appropriate reference frame and choice of inputs and outputs requires consideration. The use of the Park and Clarke $(d q)$ transformations in a common reference frame, as in [21], [24] allows transmission lines retain their natural passivity properties. If the assumption that the transmission line dynamics are instantaneous and lossless (i.e. purely inductive) can be made, the bus dynamics can be simplified using a local reference frame $^{5}$ for the $d q$ transform.

In our case, since we are considering $\mathrm{AC}$ transmission networks, such an assumption is typically justified. In this case the transmission lines are passive if these are viewed as a system with input the frequency (deviation) $\omega_{j}$ and voltage magnitude

\footnotetext{
${ }^{5}$ It should be noted that in our numerical investigations we found it not feasible to design ILC controllers such that the passivity condition on the ILC is satisfied, when a synchronous reference frame is used.
} 


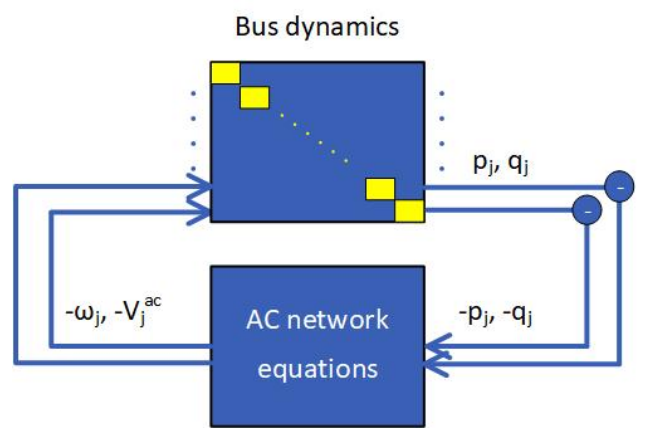

Figure 2. AC grid representation as feedback interconnection of Network/Bus subsystems.

(deviation) $V_{j}^{a c}$ of each bus $j$, i.e. $u=\left\{\omega_{1}, V_{1}^{a c}, \omega_{2}, V_{2}^{a c}, \ldots\right\}$ and output the active and reactive power $p_{j}$ and $q_{j}$, i.e. $y=\left\{p_{1}, q_{1}, p_{2}, q_{2}, \ldots\right\}$. The bus dynamics have a block diagonal structure (Fig. 2) and therefore, if the dynamics at each bus $j \in N_{a c}$ with input $-\left[\omega_{j} V_{j}^{a c}\right]^{T}$ and output $\left[p_{j} q_{j}\right]^{T}$ are strictly passive about an equilibrium point then the stability of that equilibrium point can be deduced.

\section{Hybrid AC/DC network passivity}

Fig. 3 shows how the passivity conditions for AC and DC networks may be extended to hybrid AC/DC networks. In the figure, we illustrate how the ILC dynamics couple the AC/DC dynamics and therefore its inputs and outputs involve both $\mathrm{AC}$ and DC variables. In particular, the ILC is a dynamical system with three-inputs and three-outputs as shown in Fig.

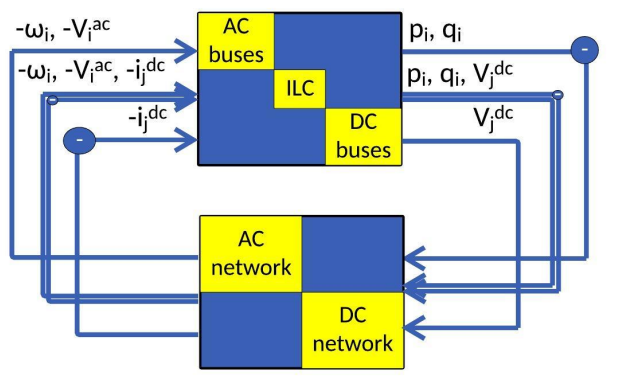

Figure 3. AC/DC grid representation as feedback interconnection of Network/Bus subsystems.

4. Therefore, the decentralized passivity condition for an ILC connecting buses $i \in X_{a c}$ and $j \in X_{d c}$ is as given in Definition 1 , i.e. we require passivity about an appropriate equilibrium point of the system with input $-\left[\begin{array}{lll}\omega_{i} & V_{i}^{a c} & i_{j}^{d c}\end{array}\right]^{T}$ and output $\left[\begin{array}{lll}p_{i}^{X} & q_{i}^{X} & V_{j}^{d c}\end{array}\right]^{T}$. A rigorous derivation of why this property leads to stability of the network will be given in the next sections. It should also be noted that the hybrid network we consider in the next sections consists of multiple ILCs and AC/DC networks (rather than only one ILC as in Figure 3).

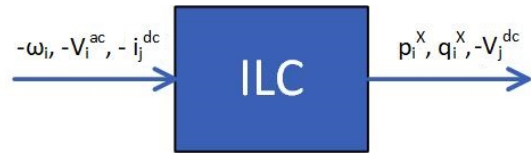

Figure 4. ILC inputs and outputs
In the rest of this paper, we consider the models of the network, buses and interlinking converters which we use for our formal stability proof. We then consider how the ILC dynamics can be designed to satisfy the decentralized passivity condition in conjunction with achieving a suitable power sharing objective.

\section{NETWORK MODEL}

\section{A. Transmission line model}

We assume that the dynamic behaviour of the transmission lines occurs on a much faster timescale than that of the network, and thus can be neglected. As stated before in section III-C, this approximation is generally valid for conventional transmission networks with synchronous generators, e.g. [23], [24], although care should be taken to verify this assumption for converter-based grids.

1) AC Power-flow model: Neglecting the AC transmission line dynamics results in the usual AC power-flow equations. We also make a further assumption and use the well-known linear AC power-flow representation (usually referred to as the DC power-flow equations; however note that this is for $\mathrm{AC}$ networks rather than DC networks) to represent power transfers across the network. Note that this can be relaxed to any power-flow model which preserves passivity from the input local active and reactive power at each bus $j$, i.e. $p_{j}, q_{j}$ to the output local frequency $\omega_{j}$ and voltage magnitude $V_{j}^{a c}$. For completeness, we also state the assumptions used in the DC power-flow approximation in Assumption 1:

\section{Assumption 1:}

- Voltage angle differences between buses remain small, such that $\sin \eta_{i j} \approx \eta_{i j}$ and $\cos \eta_{i j} \approx 1$.

- Voltage magnitudes remain close to the nominal (1 p.u), i.e. the voltage deviation $V_{j}^{a c} \ll 1$.

- The transmission lines are lossless.

Applying these assumptions to the power-flow equations results in the simplified linear power-flow equations in (3):

$$
\begin{aligned}
p_{i j} & =B_{i j} \eta_{i j} & & (i, j) \in E_{a c} \\
q_{i j} & =B_{i j}\left(V_{i}^{a c}-V_{j}^{a c}\right) & (i, j) & \in E_{a c}
\end{aligned}
$$

2) DC Power-flow model: For DC networks, reactive power is absent. The power transfer equation can be derived from Ohm's Law:

$$
i_{i j}=G_{i j}\left(V_{i}^{d c}-V_{j}^{d c}\right) \quad(i, j) \in E_{d c}
$$

where $i_{i j}$ is the branch current flow deviation. Supposing that the voltages remain close to 1 p.u., we have $p_{i j} \approx i_{i j}$.

Another notion we will use in our analysis is the total power (current) transfer from the DC-side bus of the ILC into the DC subsystem via the connected transmission lines. This is denoted by:

$$
i_{j}^{d c}=-\sum_{i: i \rightarrow j} i_{i j}+\sum_{k: j \rightarrow k} i_{j k}, j \in X_{d c}
$$

Remark 4: Note that it is possible to include resistiveinductive DC line dynamics in the model without affecting the 
passivity property of the lines. However, for simplicity as well as consistency with the AC line model, we have considered static DC lines in this paper.

\section{B. Generator model}

We use a simplified third-order generator model derived from [32], which includes voltage dynamics and allows the exciter dynamics to be modelled via the exciter emf (deviation) $E_{j}^{f d}$. The generator dynamics for a bus $j \in \mathbb{N}_{a c}$ are given by (6):

$$
\begin{aligned}
M_{j} \dot{\omega}_{j} & =-D_{j} \omega_{j}+p_{j}^{M}-p_{j}^{L}+\sum_{i: i \rightarrow j} p_{i j}-\sum_{k: j \rightarrow k} p_{j k} \\
\tau_{j}^{g} \dot{V}_{j}^{a c} & =a_{j} E_{j}^{f d}-b_{j} V_{j}^{a c}-X_{j} q_{j}^{G}
\end{aligned}
$$

where $M_{j}, D_{j}, p_{j}^{L}, \tau_{j}^{g}, a_{j}, b_{j}, X_{j} \in \mathbb{R}$ are positive constants described in Table I and $\omega_{j}, p_{i j}, V_{j}^{a c}, q_{j}^{G}, p_{j}^{M}$ are time varying quantities that take values in $\mathbb{R}$ (also described in the Table I).

Variable $p_{j}^{M}$ is the mechanical power at bus $j$, and is the output an asymptotically stable linear dynamical system (corresponding to the turbine/governor dynamics) with input the frequency deviation $\omega_{j}$. Furthermore $E_{j}^{f d}$ is the output of an asymptotically linear system with input $V_{j}^{a c}$ (and sometimes $\omega_{j}$ as well, especially if a power system stabilizer is present). To simplify our analysis we also define the following variables:

$$
\begin{aligned}
p_{j}^{G} & =p_{j}^{M}-D_{j} \omega_{j} \\
e_{j}^{G} & =\frac{1}{X_{j}}\left(a_{j} E_{j}^{f d}-b_{j} V_{j}^{a c}\right) \\
\tau_{j}^{V} & =\tau_{j}^{G} / X_{j}
\end{aligned}
$$

We now consider the overall system associated with generation at bus $j \in \mathbb{N}_{a c}$ with input $u=-\left[\omega_{j} V_{j}^{a c}\right]^{T}$ and outputs $y=\left[p_{j}^{G} e_{j}^{G}\right]^{T}$. This is a linear system of the form

$$
\begin{aligned}
\dot{x}_{j}^{S G} & =\mathcal{A}_{j} x_{j}^{S G}+\mathcal{B}_{j} u \\
y & =\mathcal{C}_{j} x_{j}^{S G}+\mathcal{D}_{j} u
\end{aligned}
$$

where $\mathcal{A}_{j} \in \mathbb{R}^{m_{j} \times m_{j}}, \mathcal{B}_{j} \in \mathbb{R}^{m_{j} \times 2}, \mathcal{C}_{j} \in \mathbb{R}^{2 \times m_{j}}, \mathcal{D}_{j} \in \mathbb{R}^{2 \times 2}$ and $x_{j}^{S G}$ is the state vector.

We state below a passivity assumption for this system.

Assumption 2: The dynamics at buses $j \in \mathbb{N}_{a c}$ with inputs $u=-\left[\begin{array}{ll}\omega_{j} & V_{j}^{a c}\end{array}\right]^{T}$ and outputs $y=\left[\begin{array}{ll}p_{j}^{G} & e_{j}^{G}\end{array}\right]^{T}$ are input strictly passive, i.e. they satisfy the condition in Definition 1.

Remark 5: Existing studies and additional numerical investigations we have carried out demonstrate that it is practically feasible for Assumption 2 to be satisfied in existing grids. In particular, in [23] it was shown that existing turbine-governor dynamics very often satisfy the passivity condition. Within this paper we have numerically examined in the same test system the passivity properties of the system described above, which includes also voltage dynamics, and found that appropriate exciter designs may allow it to satisfy the passivity property (at all frequencies). In other cases, a small reduction in droop or exciter gain allows the passivity property to be achieved.

For DC generation, simple droop control relating power and DC voltage is standard, and this satisfies the passivity assumption. We consider for generality an asymptotically stable linear system representing DC generation at a bus $j \in \mathbb{G}_{d c}$ i.e.

$$
\begin{aligned}
\dot{x}_{j}^{D G} & =\mathcal{A}_{j} x_{j}^{D G}+\mathcal{B}_{j} u \\
p_{j}^{G} & =\mathcal{C}_{j} x_{j}^{D G}+\mathcal{D}_{j} u
\end{aligned}
$$

where $\mathcal{A}_{j} \in \mathbb{R}^{m_{j} \times m_{j}}, \mathcal{B}_{j} \in \mathbb{R}^{m_{j} \times 1}, \mathcal{C}_{j} \in \mathbb{R}^{1 \times m_{j}}, \mathcal{D}_{j} \in \mathbb{R}$, $u=-V_{j}^{d c}$, and $x_{j}^{D G}$ is the state vector. We state below a passivity assumption on this system.

Assumption 3: The generator dynamics at buses $j \in \mathbb{G}_{d c}$ with input $u=-V_{j}^{d c}$ and output $p_{j}^{G}$ are input strictly passive, i.e. they satisfy the condition in Definition 1.

\section{Full network model}

For clarity, we provide the overall system equations in (10).

$$
\begin{aligned}
\dot{\eta}_{i j} & =\omega_{i}-\omega_{j}, \quad(i, j) \in E_{a c} \\
M_{j} \dot{\omega}_{j} & =p_{j}^{G}+p_{j}^{X}-p_{j}^{L}+\sum_{i: i \rightarrow j} p_{i j}-\sum_{k: j \rightarrow k} p_{j k}, \quad j \in \mathbb{N}_{a c} \\
\tau_{j}^{V} \dot{V}_{j}^{a c} & =e_{j}^{G}+q_{j}^{X}-q_{j}^{L}-\sum_{i: i \rightarrow j} q_{i j}+\sum_{k: j \rightarrow k} q_{j k}, j \in \mathbb{N}_{a c} \\
C_{j} \dot{V}_{j}^{d c} & =p_{j}^{G}-p_{j}^{L}+\sum_{i: i \rightarrow j} i_{i j}-\sum_{k: j \rightarrow k} i_{j k}, j \in \mathbb{G}_{d c} \quad(10 \mathrm{~b}) \\
p_{i j} & =B_{i j} \eta_{i j}, \quad j \in N_{a c}, \quad(i, j) \in E_{a c} \\
q_{i j} & =B_{i j}\left(V_{i}^{a c}-V_{j}^{a c}\right), \quad j \in N_{a c}, \quad(i, j) \in E_{a c} \quad(10 \mathrm{~d}) \\
i_{i j} & =G_{i j}\left(V_{i}^{d c}-V_{j}^{d c}\right), \quad j \in N_{d c}, \quad(i, j) \in E_{d c} \quad(10 \mathrm{~g})
\end{aligned}
$$

The system equations may be described as follows:

- Equations (10a)-(10b) are the third-order generator models, where variables $p_{j}^{G}, e_{j}^{G}$ are as defined in (7), (8) and the preceding text.

- (10d) is the DC voltage equation, where the DC generation $p_{j}^{G}$ satisfies (9).

- (10e)-(10g) are the power-flow equations.

- $p_{j}^{X}, q_{j}^{X}$ are time varying quantities denoting real/reactive power transfers associated with the ILC (see Table I). Dynamics associated with the ILC will be described in the next section.

\section{INTERLINKING CONVERTER CONTROL}

In this section we present the problem considered in this paper, namely the design of the interlinking converter control scheme so as to have network stability and appropriate powersharing. We start by presenting the control objectives, followed by a stability result for the network when the ILC satisfies an appropriately formulated decentralized passivity condition. A short review and assessment of the ILC control schemes proposed in literature is then given, to see whether they can achieve the criterion specified, while achieving a desired steady-state power allocation. Finally, we discuss inherent trade-offs and propose an ILC control scheme that achieves the desired objectives.

Control objectives: 
1) Stability: Provide stability guarantees for the network by means of local conditions on the bus dynamics.

2) Power sharing: The power transfer between the AC and $D C$ subsystems should be such that the droop-controlled $A C$ and DC sources contribute in proportion to their droop coefficients. This in turn requires establishing a prescribed relation between the AC frequencies and the $D C$ voltages at steady state, since these are the variables used for droop control.

These two control objectives are related by the fact that the power-sharing will be determined by the steady state response of the dynamics. In particular, certain control architectures which give good power sharing properties might not necessarily have good stability properties, or vice versa. Our analysis focuses on the ability of ILC control architectures to meet both objectives.

\section{A. Stability result}

We now state in Assumption 4 a decentralized passivity condition for the ILCs (note that this is analogous to that discussed in section III-D).

For our analysis we assume that the ILC dynamics are linear. In particular, for each ILC $k \in X$ connecting bus $i=X_{a c}^{k}$ to bus $j=X_{d c}^{k}$ the dynamical system with input $-\left[\omega_{i} V_{i}^{a c} i_{j}^{d c}\right]^{T}$ and output $\left[\begin{array}{lll}p_{i}^{X} & q_{i}^{X} & V_{j}^{d c}\end{array}\right]^{T}$ is linear and asymptotically stable with state vector $x_{k}^{C}$.

Assumption 4: For each ILC $k \in X$ connecting bus $i=X_{a c}^{k}$ to bus $j=X_{d c}^{k}$ the dynamical system with input $-\left[\begin{array}{llll}\omega_{i} & V_{i}^{a c} & i_{j}^{d c}\end{array}\right]^{T}$ and output $\left[\begin{array}{lll}p_{i}^{X} & q_{i}^{X} & V_{j}^{d c}\end{array}\right]^{T}$ is passive, i.e. it satisfies the condition in Definition 1.

We now present the stability result for the hybrid AC/DC network under the passivity assumption stated above. Our stability result is given in Theorem 1 and the proof is provided in the Appendix, showing that all solutions of the system converge to an equilibrium point. Note that although the convergence result is global (due to the linear power transfer dynamics (10e) and the assumption of linear converter/generation dynamics), the linearizing assumption for the transmission lines (Assumption 1) is only valid for small angle differences between buses and hence in practice the stability property stated is a local one. Note also that the linearity assumption for the ILC dynamics could be relaxed as we will discuss in Remark 6.

Theorem 1 (Stability): Consider a dynamical system described by equations (10), with $\mathrm{AC}$ and $\mathrm{DC}$ generation and ILC dynamics satisfying Assumptions 2, 3 and 4 respectively. Then all solutions of (10) converge to an equilibrium point.

Remark 6: It should be noted that the stability proof in the appendix is Lyapunov based. If the system has non-linear dynamics for which the passivity property in Definition 1 holds about an appropriate equilibrium point, local convergence to an equilibrium point can also be deduced using an analogous Lyapunov function. Note that the Lyapunov function used to prove stability consists of the storage functions of the $\mathrm{AC}$ network, the DC network, and the ILC.
Theorem 1 shows that if decentralized passivity conditions are satisfied, stability of the network can be deduced. As discussed at the beginning of this section, in addition to stability our desired objectives include also achieving satisfactory power sharing. In the following section we provide analysis and simulations to illustrate various underlying trade-offs and demonstrate the applicability of our results for ILC control design.

\section{B. Converter models}

Since transmission networks operate slowly compared to the internal dynamics of the converter, we model the interlinking converter as either first-order controllable voltage sources, or first-order controllable power sources with some time constant $\tau_{i}^{X}$. The first-order time constant representation is typical for simplified models of converters in power grids, e.g. [33], and are relevant for the study of grid dynamics at medium to slower timescales. The distinction between setting the ACside voltage and frequency (voltage control) or controlling the power transfer directly (power control) is analogous to that between grid-forming control and grid-supporting control in an AC microgrid [34]. Note that more detailed models which include hierarchical inner loops and switching dynamics are used for the simulations carried out in section VI. We illustrate our ILC notation in Fig. 5 and a detailed description of various ILC control schemes is provided in the next section.

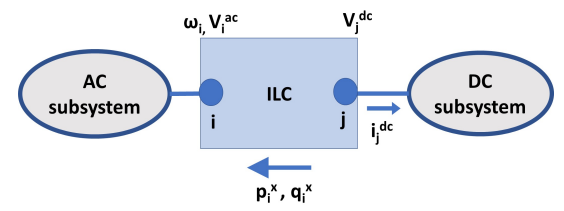

Figure 5. ILC notation.

\section{Controller review}

1) Dual-droop control schemes: In this section we consider schemes which track a power setpoint determined by the AC frequency and DC voltage, namely conventional dual droop control [9], and normalized integral dual-droop control [10][13]. The model of the ILC in dual droop is (11):

$$
\begin{aligned}
\tau_{i}^{x} \dot{q}_{i}^{X} & =-q_{i}^{X}-n_{i}^{-1} V_{i}^{a c} \\
\tau_{i}^{x} \dot{p}_{i}^{X} & =-p_{i}^{X}-K_{i}^{\omega} \omega_{i}+K_{i}^{V} V_{j}^{d c} \\
C_{j} \dot{V}_{j}^{d c} & =-p_{i}^{X}-i_{j}^{d c}-K_{j}^{d c} V_{j}^{d c}
\end{aligned}
$$

where constants $K_{i}^{\omega}$ and $K_{i}^{V}$ are droop coefficients, and constant $K_{i}^{I}$ is the integral gain. The DC current into the ILC from the network, $-i_{j}^{d c}$, is defined in (5) (similarly for the other converter models). Similarly, the model of the ILC in normalized integral dual-droop mode is:

$$
\begin{aligned}
\dot{\xi}_{i} & =-K_{i}^{\omega} \omega_{i}+K_{i}^{V} V_{j}^{d c} \\
\tau_{i}^{x} \dot{q}_{i}^{X} & =-q_{i}^{X}-n_{i}^{-1} V_{i}^{a c} \\
\tau_{i}^{x} \dot{p}_{i}^{X} & =-p_{i}^{X}-K_{i}^{\omega} \omega_{i}+K_{i}^{V} V_{j}^{d c}+K_{i}^{I} \xi_{i} \\
C_{j} \dot{V}_{j}^{d c} & =-p_{i}^{X}-i_{j}^{d c}-K_{j}^{d c} V_{j}^{d c}
\end{aligned}
$$


2) Matching ILC control: This controller, inspired by [18], was proposed in our previous work [14], where the analysis focused on the power sharing properties with simpler static models used for inverters. Let the frequency at the ILC (internal) AC bus be set as:

$$
\omega_{i}^{X}=m V_{j}^{d c}
$$

This relates the AC frequency deviation $\omega_{i}^{X}$ proportionally to the DC voltage deviation $V_{j}^{d c}$ by a chosen constant $m>0$. Note that the same equilibrium is reached in (12) with $m=K_{i}^{V} / K_{i}^{\omega}$. A key feature of this control scheme is to note that rather than controlling the power transfer, this controller directly regulates the AC-side frequency and voltage. The power injection $p_{i}^{X}$ is instead determined by the angle difference and impedance between the internal $\mathrm{AC}$ bus of the ILC with frequency $\omega_{i}$ in (13) and the bus of the AC subsystem to which the ILC is connected. The model of the ILC in matching control mode is:

$$
\begin{aligned}
\dot{\eta}_{i}^{X} & =-\omega_{i}+m_{i} V_{j}^{d c} \\
\tau_{i}^{x} \dot{V}_{i}^{a c X} & =-V_{i}^{a c X}-n_{i}^{-1} q_{i}^{X} \\
C_{j} \dot{V}_{j}^{d c} & =-p_{i}^{X}-i_{j}^{d c}-K_{j}^{d c} V_{j}^{d c} \\
p_{i}^{X} & =B_{i} \eta_{i}^{X} \\
q_{i}^{X} & =B_{i}\left(V_{i}^{a c X}-V_{i}^{a c}\right)
\end{aligned}
$$

where $\eta_{i}^{X}$ is the angle difference between the internal AC bus (i.e. the converter side of the AC filter) of the ILC and the bus of the AC subsystem to which the ILC is connected, $V_{i}^{a c X}$ is the voltage magnitude deviation of the internal $\mathrm{AC}$ bus of the ILC, and $B_{i}>0$ is the magnitude of the filter susceptance.

\section{Control design of interlinking converters}

As discussed in section $\mathrm{V}$, we consider conventional dual droop control [9], normalized integral dual-droop control [10]-[13], and controller based on matching control recently proposed in our previous work [14]. The simplified models may be easily assessed to determine whether appropriate parameter choices can satisfy the passivity condition, and for completeness, we also numerically examine more detailed full order converter models which include internal voltage and current loops.

For the numerical evaluation, we adopt the following approach. As stated in Remark 3, passivity requires the positive realness of the corresponding transfer function matrix which we denote $H(s)$. Therefore, in order to satisfy the condition the eigenvalues of the Hermitian part of the transfer matrix at all frequencies must be non-negative, i.e. $H(j \omega)+H^{*}(j \omega) \geq 0$. This is easy to examine numerically and it becomes possible to identify the frequency ranges at which passivity may be lacking. The numerical evaluation may be described as follows, and is also summarized in Table II:

- The dual-droop controlled ILC is the controller for which we were able to find reasonable parameters to satisfy the passivity condition. However, as stated earlier there are power-sharing issues.

- The dynamics of integral dual-droop are passive for most frequencies, however at some frequency ranges the transfer matrix does not satisfy $H(j \omega)+H^{*}(j \omega) \geq 0$. This range can be reduced if the gains are small, but in our investigations we were not able to achieve passivity by tuning the gains.

- For matching control, the simple model presented in (14) could not be passivated ${ }^{6}$, although the power sharing objective is achieved.

Table II

PASSIVITY SUMMARY: EXISTING SCHEMES

\begin{tabular}{c|c|c} 
Controller & Passivity assessment & Power sharing \\
\hline Dual-droop & Can be passive & Average \\
Integral droop & Difficult to passivate & Good \\
Matching & Difficult to passivate & Good
\end{tabular}

It is clearly difficult to achieve both control objectives simultaneously: guaranteed stability via decentralized conditions, and acceptable power sharing. The dual-droop controllers achieve one objective or the other, but not both simultaneously. The matching controller in (14) is also unable to satisfy the passivity condition. We therefore consider an alternative control design to achieve both properties satisfactorily.

In designing a controller to achieve power-sharing with droop controlled sources, it is imperative to equalize the normalized AC frequency and DC voltage, i.e. at steadystate $\omega_{i}=m_{i} V_{j}^{d c}$. For this reason, the decentralized ILC control schemes in the literature achieving good power sharing performance have aimed for this relation at equilibrium, e.g. [13], [14]. This allows the AC frequency / DC voltage to act as a synchronizing variable for the droop-controlled AC and DC sources, thus achieving optimal power sharing if line resistances are small [10], [13], [14].

However, a necessary (but not sufficient) condition to achieve passivity is that the steady state input-output map must be passive. Equivalently, the transfer function $H$ of the ILC satisfies $H(0)+H^{T}(0) \geq 0$. Controlling the power of the ILC such that at steady state the relationship $\omega_{i}=m_{i} V_{j}^{d c}$ holds, results in a zero on the diagonal of $H(0)+H^{T}(0)$ and a positive off diagonal term. Therefore $H(0)+H^{T}(0)$ cannot be positive semidefinite since it has a principal $2 \times 2$ submatrix with negative determinant (see e.g. [35, chapter 7.1]). This reveals the existence of a fundamental dilemma: to achieve power sharing by means of equalizing AC frequency and DC voltage results in a lack of passivity at low frequencies. A fundamental trade-off exists between these two goals of achieving passivity and therefore stability guarantees, and achieving a prescribed power sharing via $\omega_{i}=m_{i} V_{j}^{d c}$. This trade-off can be improved by designing the transfer matrix to be as close as possible to skew-symmetric, however in practice it cannot be completely avoided.

Taking into account this trade-off we propose a control design that allows a slight inaccuracy in power sharing to

\footnotetext{
${ }^{6}$ Using an even simpler model (which models the converter filter as part of the network rather than the ILC), it is possible to achieve passivity (but not strict passivity). However, this requires exact gains (which are difficult to achieve practically) and passivity cannot be achieved when more advanced models are used. Hence this is not a practical control design to satisfy the passivity condition.
} 
achieve stability guarantees via the passivity property. In particular, we propose a small modification of the integral dual-droop scheme. By using a leaky integrator instead of a pure integrator (i.e. a first-order lag term with a very low cutoff frequency), passivity may be achieved by appropriately tuning the gains. In particular, an additional $-\epsilon_{i} \xi$ term is added in the righthand side of (12a) (see (15a)). This term causes a small error in the power-sharing, however this is not substantial (1\% or less). Another way to address this tradeoff is by having the ILC close enough to a droop-controlled source such that an appropriate diagonal term in $H(0)$, to help achieve passivity, can be provided by the local source. This is consistent with the practical intuition that AC/DC connections should be made at strong parts of the grid.

We also further improve transient performance by the addition of a suitable lead compensator of the standard form $\frac{\alpha s+\beta}{\alpha^{-1} s+\beta}$ with parameters $\alpha>1$ and $\beta>0$ which control the shape of the phase lead and the frequency at which it is applied respectively. The lead compensator preserves the desired passivity property and is placed at the controller output associated with active power $p_{i}^{X}$ in the ILC. For completeness, we provide the amended ILC equations in (15):

$$
\begin{aligned}
\dot{\xi}_{i} & =-K_{i}^{\omega} \omega_{i}+K_{i}^{V} V_{j}^{d c}-\epsilon_{i} \xi_{i} \\
\tau_{i}^{x} \dot{q}_{i}^{X} & =-q_{i}^{X}-n_{i}^{-1} V_{i}^{a c} \\
\dot{x}_{i} & =-\alpha_{i} \beta_{i} x_{i}+\left(-K_{i}^{\omega} \omega_{i}+K_{i}^{V} V_{j}^{d c}+K_{i}^{I} \xi_{i}\right) \\
\tau_{i}^{x} \dot{p}_{i}^{X} & =-p_{i}^{X}-\alpha_{i} \beta_{i}\left(\alpha_{i}^{2}-1\right) x_{i} \\
& +\alpha_{i}^{2}\left(-K_{i}^{\omega} \omega_{i}+K_{i}^{V} V_{j}^{d c}+K_{i}^{I} \xi_{i}\right) \\
C_{j} \dot{V}_{j}^{d c} & =-p_{i}^{X}-i_{j}^{d c}-K_{j}^{d c} V_{j}^{d c}
\end{aligned}
$$

where $x_{i}$ is the state associated with the compensator. The passivity property is satisfied for suitable parameter choices as we will illustrate shortly.

We demonstrate below the passivity properties of the proposed controllers in the frequency domain. Since passivity is equivalent to the positive realness of the corresponding transfer function, the eigenvalues of the Hermitian part of the transfer matrix at all frequencies must be non-negative, i.e. we require $H(j \omega)+H^{*}(j \omega) \geq 0$. We can therefore deduce the passivity property via the eigenvalues of this Hermitian matrix over frequency. Fig. 6 compares the eigenvalues of this matrix for the three control strategies over a range of frequencies, using the models presented in (11), (12), (14), (15). Parameter values for all models are as follows: $K_{i}^{\omega}=2.5 \times 10^{5} \mathrm{~W} /(\mathrm{rad}$ $\left.s^{-1}\right), K_{i}^{V}=5 \times 10^{2} \mathrm{~W} / \mathrm{V}, \epsilon_{i}=10^{-2}, \tau_{i}^{x}=0.05 \mathrm{~s}$, $n_{i} \geq 0, K_{i}^{I}=0.1, C_{j}=10^{-3} \mathrm{mF}, \alpha_{i}=2, \beta_{i}=2 \times 10^{-2}$, $K_{j}^{d c}=1 \mathrm{~W} / \mathrm{V}, m_{i}=0.002, B_{i}=3.18$.

In this numerical study we considered an extensive range of parameters and compensators for each ILC controller, however we were unable to achieve passivity for some control architectures. As discussed, this is because their steadystate input/output map is non-passive. The lack of passivity associated with the non-passive steady-state input/output map occurs by definition for low frequencies, which corresponds

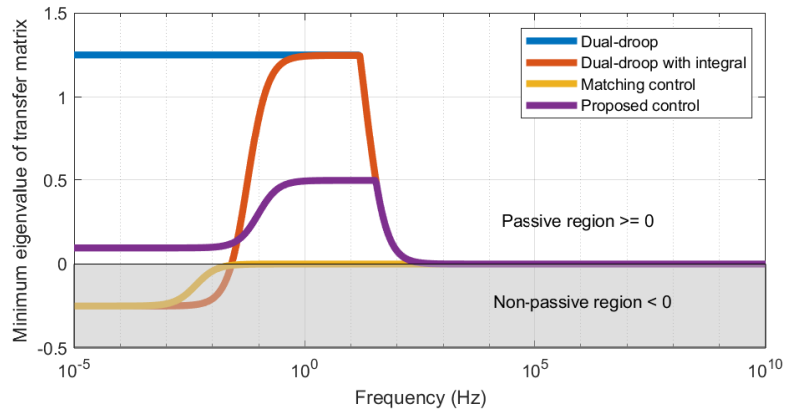

Figure 6. Passivity analysis of interlinking converters (11), (12), (14), (15).

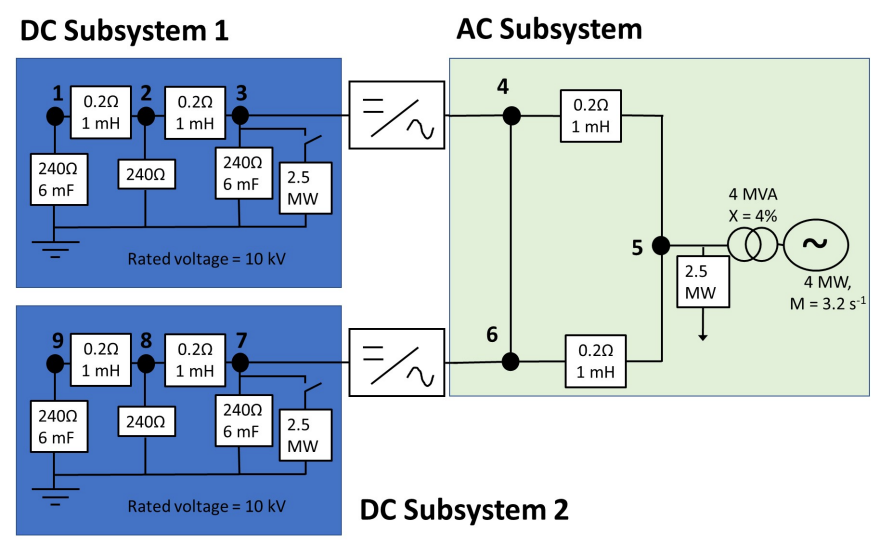

Figure 7. AC/DC network diagram.

to a risk of low-frequency stability issues (such as inter-area oscillations) if the passivity condition is not satisfied.

\section{CASE STUdY AND DISCUSSION}

We perform simulations on the simple test hybrid AC/DC network in Figure 7 (also used in [14]), in order to verify our analysis. The parameters of the network are given in Table III. A 4 MVA, $13.8 \mathrm{kV}$ synchronous machine is connected to the AC subsystem at bus 5 via a 4 MVA step-down transformer, and there are four distributed DC generators across the two subsystems at buses 1 and 9 ( $1 \mathrm{MW}$ ) and buses 3 and 7 (3 MW). The simulation model is more detailed and realistic than our analytical model, and it includes the converter dynamics with switching (two-level pulse width modulation), hierarchical inner-loop dynamics, the LCL filter dynamics, transmission line dynamics, detailed generator, turbine-governor and exciter dynamics, and realistic communication delays ${ }^{7}$. The droop coefficients are set proportionally to the source ratings with gains of $(500 \mathrm{~kW} /(\mathrm{rad} / \mathrm{s}), 1 \mathrm{~kW} / \mathrm{V}, 3 \mathrm{~kW} / \mathrm{V})$ for the synchronous generator, DC sources at buses 1 and 9, DC sources at buses 3 and 7 respectively.

In Figs. 8-13 we compare the performance of the three decentralized ILC controllers considered in this paper. We show the AC frequency and DC voltage response to the same

\footnotetext{
${ }^{7}$ Further details, including the equilibrium points in the simulation, are also available at: https://doi.org/10.13140/RG.2.2.20682.39367/1
} 
Table III

HYBRID AC/DC NETWORK PARAMETERS

\begin{tabular}{c|c|c} 
Description & Parameter/Location & Value \\
\hline Bus capacitances & $C_{1}, C_{3}, C_{7}, C_{9}$ & $6 \mathrm{mF}$ \\
DC load resistances & $R_{j}, j \in N_{d c}$ & $600 \Omega$ \\
DC line resistances & $R_{12}, R_{23}, R_{78}, R_{89}$ & $0.2 \Omega$ \\
DC line inductances & $L_{12}, L_{23}, L_{78}, L_{89}$ & $1 \mathrm{mH}$ \\
DC switched loads & $p_{3}, p_{7}$ & $1.2 \mathrm{MW}$ \\
Rated DC voltage & $V^{d c}$ & $10 \mathrm{kV}$ \\
DC source ratings & (Buses 1 and 9) & $1 \mathrm{MW}$ \\
DC source ratings & Buses 3 and 7) & $3 \mathrm{MW}$ \\
Converter DC capacitance & $C_{3}, C_{7}$ & $2 \mathrm{mF}$ \\
Converter filter parameters & $R, L, C$ & $1 \mathrm{mH}, 10 \mu F$ \\
Converter $\omega /$ ratio & $m$ & 0.002 \\
Rated AC voltage & $V^{a c}$ & $3.3 \mathrm{kV}$ \\
AC line resistances & $R_{i j}$ & $0.2 \Omega$ \\
AC line inductances & $L_{i j}$ & $1 \mathrm{mH}$ \\
AC load resistances & $R_{4}, R_{6}$ & $1000 \Omega$ \\
AC load active power & $R_{5}$ & $1 \mathrm{MW}$ \\
Transformer reactance & $X_{5}$ & $4 \%$ \\
Generator inertia constant & $M_{5}$ & $3.2 s^{-1}$ \\
AC rated frequency & $f=\frac{\omega}{2 \pi}$ & $50 \mathrm{~Hz}$ \\
\hline DC source droop gains & (Buses 1 and 9) & $1 \mathrm{~kW} / \mathrm{V}$ \\
DC source droop gains & (Buses 3 and 7$)$ & $3 \mathrm{~kW} / \mathrm{V}$ \\
Generator droop gain & $(B u s 5)$ & $500 \mathrm{~kW} /(\mathrm{rad} / \mathrm{s})$ \\
ILC dual-droop gains & DC side & $500 \mathrm{~W} / \mathrm{V}$ \\
ILC leaky integrator term & AC side & $250 \mathrm{~kW} /(\mathrm{rad} / \mathrm{s})$ \\
ILC compensator terms & $\epsilon$ & 0.01 \\
ILC matching constant & $\alpha, \beta$ & $10^{2}, 10^{-2}$ \\
& $m$ & 0.002 \\
& &
\end{tabular}

step disturbances at time $t=3 \mathrm{~s}$ and $t=23 \mathrm{~s}$. The magnitude of the disturbance at $t=3 \mathrm{~s}$ is $1.2 \mathrm{MW}$ (nominal added demand) located at bus 3 within DC subsystem 1, while the disturbance $t=23 \mathrm{~s}$ is $1.2 \mathrm{MW}$ reduced demand at bus 7 within DC subsystem 2. The traditional dual-droop controller is used in Figs. 8-9 where we note that it successfully stabilizes the network.

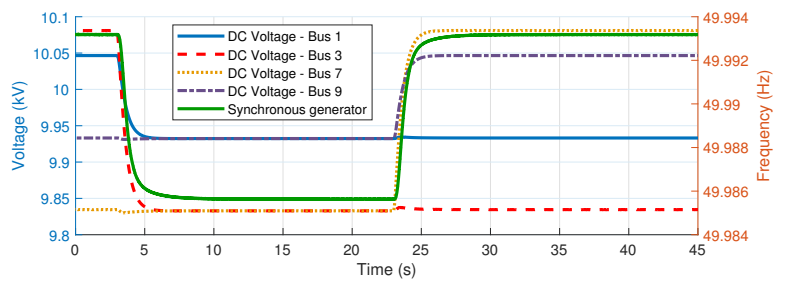

Figure 8. Frequency and voltage response with the dual-droop ILC controller (12)

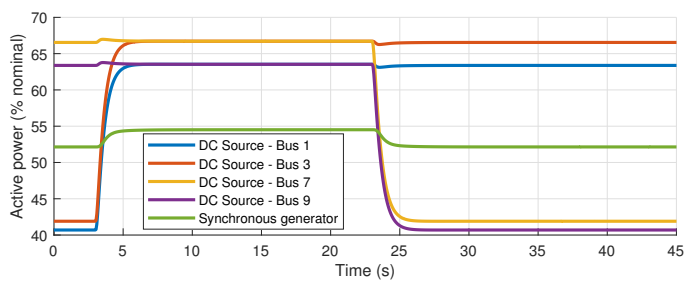

Figure 9. Active power of the dual-droop ILC controller (11). The value at steady state demonstrates the power-sharing.

In order to improve the power sharing performance, two approaches have been proposed. The first adds an integral term to the dual-droop controller, and allows power sharing to be

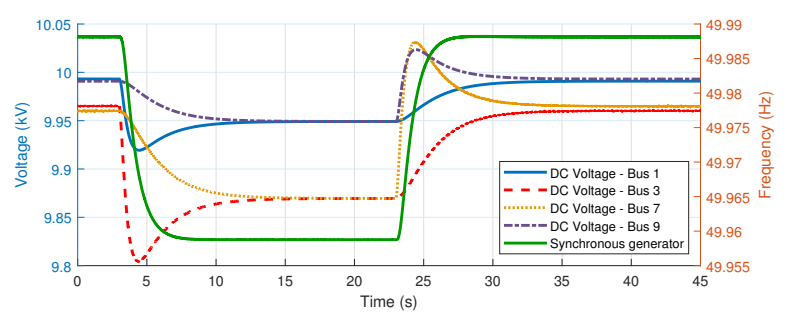

Figure 10. Frequency and voltage response with the leaky integral dual-droop ILC controller (15)

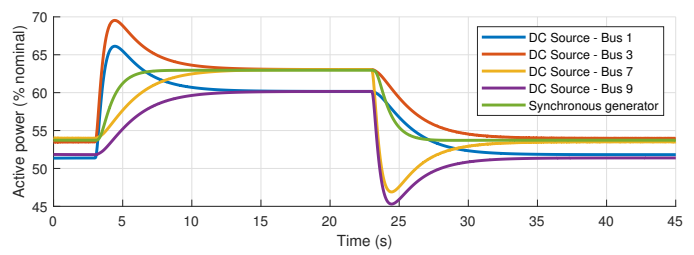

Figure 11. Active power with the leaky integral dual-droop ILC controller (15). The value at steady state demonstrates the power-sharing.

improved by relating at steady state the frequencies of the AC subgrids to the voltages of the DC subgrids. This allows droop control schemes at the generation sources to achieve a prescribed power sharing if line resistances are small [10], [13], [14], [36]. In order to have guaranteed stability and scalability via passivity, and to improve performance, we use the leaky integrator and phase lead compensator proposed in this paper. Figs. 10-11 show that both power-sharing and good transient performance are achieved. The powers converge to similar values unlike the traditional dual-droop controller in Fig. 9. Also the error resulting from the use of the leaky integrator is negligible.

The other approach to achieve power sharing in the primary time-frame is the matching ILC controller proposed in our previous work [14]. Figs. 10-11 show that the powers do converge to values comparable to those obtained when the integral dual-droop controller is used (Fig. 11), again representing a significant improvement on the traditional scheme in Fig. 9. However, in practice transient performance is highly dependent on the presence of enough DC capacitance, requiring either low gains (slower response) or significant DC capacitance to reduce the DC voltage overshoot, and therefore the frequency overshoot, to acceptable values. A further consideration is the DC ripple, which depends on both the DC capacitance and converter topology. This will be reflected in the AC frequency and must therefore be reduced to acceptable levels. Furthermore, as explained in section V-D it is not possible to achieve the passivity property on the ILC in this case making stability guarantees difficult to achieve.

Simulations show that matching control may be destabilized by an excessively high matching gain, while similarly a large integral droop gain in the integral dual-droop scheme results in oscillatory behaviour. It should be noted that these gains affect the passivity property of the ILC considerably, whereas stability is ensured when the passivity property is satisfied. The simulations above demonstrate that the dual droop controller with a leaky integrator term, lead compensator, combined 


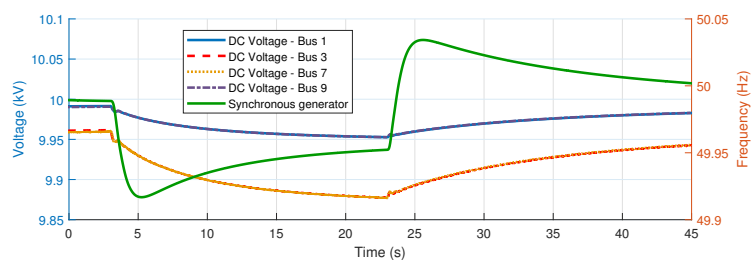

Figure 12. Frequency and voltage response with the matching ILC controller (13)

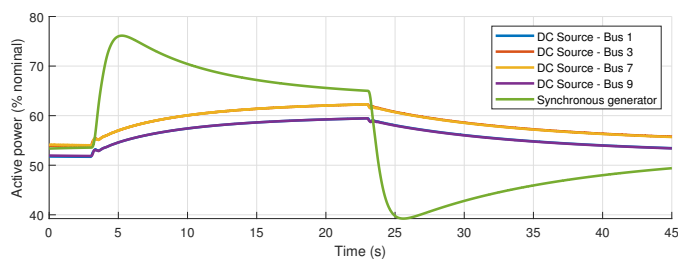

Figure 13. Active power with the matching ILC controller (13).

with reactive power - voltage droop provides an appropriate control design for hybrid AC/DC transmission systems. Both the transient performance and the steady-state power-sharing objectives are met, and its inherent passive behaviour allows an analytical guarantee of stability within the hybrid network.

\section{CONCLUSION}

This paper has considered the problem of designing controllers for interlinking converters in hybrid AC/DC grids to ensure network stability and share power efficiently between $\mathrm{AC}$ and DC sources. Using a passivity framework, we have examined existing ILC control schemes and proposed modifications such that an appropriately formulated decentralized passivity condition is satisfied, which allows to guarantee stability while retaining good performance and power-sharing properties. We have presented a case study and simulation results to show that the proposed passivity framework is applicable for control design in hybrid AC/DC networks. We have shown that the frequency and voltages of the hybrid AC/DC network can be regulated in such a way to satisfy the passivity condition and ensure stability, while also achieving good transient responses and appropriate power sharing. Future work can include: the experimental demonstration of a scaled AC/DC grid, the development of new and improved controllers for the ILC, the application of passivity approaches to converter-based hybrid AC/DC grids with more advanced models for the converters and their interconnections, and the application of passivity theory to secondary-control designs for hybrid AC/DC grids.

\section{APPENDIX}

The appendix includes the proof of the stability result presented in the main text (Theorem 1). We provide first some notation that will be used within the proof. The vector of angle differences is denoted as $\eta=\left[\eta_{i j}\right]_{(i, j) \in E_{a c}}$, the vector of $\mathrm{AC}$ frequency deviations from its nominal value (50 or $60 \mathrm{~Hz})$ is denoted by $\omega=\left[\omega_{j}\right]_{j \in N_{a c}}$, the vector of AC voltage deviations from their nominal value is denoted by $V^{a c}=$ $\left[V_{j}^{a c}\right]_{j \in N_{a c}}$, and the vector of DC voltage deviations is denoted by $V^{d c}=\left[V_{j}^{d c}\right]_{j \in \mathbb{G}_{d c}}$ and similarly denote $i^{d c}=\left[i_{j}^{d c}\right]_{j \in X^{d c}}$. The frequencies at the $\mathrm{AC}$ generator buses are denoted by $\omega^{G}=\left[\omega_{j}\right]_{j \in \mathbb{N}_{a c}}$. Similarly, we collect the internal states by $x^{S G}=\left[x_{j}^{S G}\right]_{j \in G_{a c}}, x^{D G}=\left[x_{j}^{D G}\right]_{j \in \mathbb{G}_{d c}}, x^{C}=\left[x_{j}^{C}\right]_{j \in X}$. The vector of the states of the interconnection is denoted by $x=\left[\begin{array}{ll}\eta \omega^{G} V^{a c} V^{d c} x^{S G} x^{D G} x^{C}\end{array}\right]$.

\section{A. Proof of Theorem 1}

We prove Theorem 1 by finding a suitable Lyapunov function $W(x)$ for the system (10). Consider an equilibrium point $x^{*}=\left(\eta^{*}, \omega^{G *}, V^{a c *}, V^{d c *}, x^{S G *}, x^{D G *}, x^{C *}\right)$ and let $W_{j}^{S G}, W_{j}^{D G}, W_{j}^{C}$ be the corresponding storage functions associated with AC generation, DC generation and the ILC when the passivity properties specified in the Theorem are satisfied. We now consider the following candidate Lyapunov function:

$$
\begin{aligned}
& \begin{array}{r}
W(x)=W\left(\eta, \omega^{G}, V^{a c}, V^{d c}, x^{S G}, x^{D G}, x^{C}\right)=W_{E}+W_{\omega} \\
\quad+W_{a c}+W_{d c}+W_{S G}+W_{D G}+W_{C}
\end{array} \\
& \begin{array}{r}
\text { where } \\
W_{E}=\frac{1}{2} \sum_{(i, j) \in E_{a c}} B_{i j}\left(\eta_{i j}-\eta_{i j}^{*}\right)^{2}, W_{\omega}=\frac{1}{2} \sum_{j \in \mathbb{N}_{a c}} M_{j}\left(\omega_{j}-\omega_{j}^{*}\right)^{2}
\end{array} \\
& \begin{array}{c}
W_{a c}=\frac{1}{2} \sum_{j \in \mathbb{N}_{a c}} \tau_{j}^{V}\left(V_{j}^{a c}-V_{j}^{a c *}\right)^{2}, W_{d c}=\frac{1}{2} \sum_{j \in \mathbb{G}_{d c}} C_{j}\left(V_{j}^{d c}-V_{j}^{d c *}\right)^{2} \\
W_{S G}=\sum_{j \in \mathbb{N}_{a c}} W_{j}^{S G}\left(x_{j}^{S G}\right), W_{D G}=\sum_{j \in \mathbb{G}_{d c}} W_{j}^{D G}\left(x_{j}^{D G}\right),
\end{array} \\
& W_{C}=\sum_{j \in X} W_{j}^{C}\left(x_{j}^{C}\right)
\end{aligned}
$$

By taking the time derivative, i.e. $\dot{W}=\frac{d W}{d t}=\frac{\partial W}{\partial \eta} \frac{d \eta}{d t}+$ $\frac{\partial W}{\partial \omega^{G}} \frac{d \omega^{G}}{d t}+\frac{\partial W}{\partial V^{a c}} \frac{d V^{a c}}{d t}+\frac{\partial W}{\partial V^{d c}} \frac{d V^{d c}}{d t}+\frac{d W^{S G}}{d t}+\frac{d W^{D G}}{d t}+\frac{d W^{C}}{d t}$, adding appropriate terms corresponding to the equilibrium conditions of (10), recalling the appropriate assumptions on the generator dynamics (Assumptions 2 and 3) and ILC dynamics (Assumptions 4), and simplifying, we can obtain:

$$
\begin{aligned}
\dot{W} & \leq \sum_{j \in \mathbb{N}_{a c}}\left(\omega_{j}-\omega_{j}^{*}\right)\left(p_{j}^{G}-p_{j}^{G *}\right)+\sum_{j \in \mathbb{N}_{a c}}\left(V_{j}^{a c}-V_{j}^{a c *}\right)\left(e_{j}^{G}-e_{j}^{G *}\right) \\
& +\sum_{j \in \mathbb{G}_{d c}}\left(V_{j}^{d c}-V_{j}^{d c *}\right)\left(p_{j}^{G}-p_{j}^{G} *\right)+\sum_{j \in X_{a c}}\left(\omega_{j}-\omega_{j}^{*}\right)\left(p_{j}^{X}-p_{j}^{X *}\right) \\
& +\sum_{j \in X_{a c}}\left(V_{j}^{a c}-V_{j}^{a c *}\right)\left(q_{j}^{X}-q_{j}^{X *}\right)+\sum_{j \in X_{d c}}\left(V_{j}^{d c}-V_{j}^{d c *}\right)\left(i_{j}^{d c}-i_{j}^{d c *}\right) \\
& +\sum_{j \in \mathbb{N}_{a c}} \dot{W}_{j}^{S G}+\sum_{j \in \mathbb{G}_{d c}} \dot{W}_{j}^{D G}+\sum_{j \in X} \dot{W}_{j}^{C}
\end{aligned}
$$

Using Assumptions 2, 3 and 4 respectively, we can show that $\mathrm{W}$ is a non-increasing function of time. $W_{E}$ has a strict global minimum at $\eta=\eta^{*}$. Likewise $W_{\omega}, W_{a c}, W_{a c}, W_{d c}, W^{S G}$, $W^{D G}, W^{C}$, have strict global minima at $\omega^{G *}, V^{a c *}, V^{d c *}$, $x^{S G *}, x^{D G *}$, and $x^{C *}$ respectively and are also quadratic (see Remark 2). Therefore $W(x)$ is radially unbounded.

We then apply LaSalle's Theorem [29], with $W$ as the Lyapunov-like function, which states that all trajectories of the system starting within a compact positively invariant set $T$ converge to the largest invariant set $\Xi$ within $T$ that satisfies $\dot{W}=0$. The latter implies from (16) using the input strict passivity of the buses in $\mathbb{N}_{a c}$ and $\mathbb{G}_{d c}$ that $\omega=\omega^{*}$, $V^{a c}=V^{a c *}, V^{d c}=V^{d c *}$. From (5), we also have that $i^{d c}$ is equal to is equilibrium value $i^{d c *}$ since $i^{d c}$ is a linear function of the voltages $V^{d c}$. Therefore, the inputs to the ILCs (i.e. 
$-\left[\omega_{i} V_{i}^{a c} i_{j}^{d c}\right]$ for each corresponding $i \in X_{a c}$ and $j \in X_{d c}$ are equal, within $\Xi$, to constant equilibrium values. Since the ILC dynamics are asymptotically stable, the ILC states $x^{C}$ are also equal to constant equilibrium values for any trajectory in the compact invariant ${ }^{8}$ set $\Xi$. Likewise $x^{S G}$ and $x^{D G}$ are constant within $\Xi$ by the asymptotic stability of the subsystems associated with generation. Also, $\omega=\omega^{*}$ implies the angle differences $\eta$ are constant within $\Xi$. The set $T$ can be chosen as $T=\left\{x: W(x)-W\left(x^{*}\right) \leq c\right\}$ for some constant $c>0$. Since $W(x)$ is radially unbounded $T$ can be chosen arbitrarily large by choosing $c$ sufficiently large, hence we have by LaSalle's Theorem [29] global convergence to the set of equilibrium points.

This can be strengthened to convergence of each trajectory to an equilibrium point using [37, Theorem 4.20], by noting that each equilibrium point is also Lyapunov stable. In particular, Lyapunov stability of $x^{*}$ follows from the fact that the positively invariant $T$ is a neighbourhood of $x^{*}$ that can be chosen sufficiently close to $x^{*}$ for $c>0$ sufficiently small. Furthermore, Lyapunov stability of any other equilibrium $\tilde{x}^{*}$ can be deduced using the same analysis ${ }^{9}$ but with $x^{*}$ replaced by $\tilde{x}^{*}$.

\section{REFERENCES}

[1] D. Jovcic and K. Ahmed, Two-Level PWM VSC HVDC Modelling, Control and Dynamics, Wiley 2015.

[2] D. Jovcic et al, "Feasibility of DC Transmission Networks," 2011 IEEE Conference on Innovative Smart Grid Technologies Europe (ISGT Europe), 5-7 Dec. 2011.

[3] K. Uhlen, A. G. Endegnanew and T. M. Haileselassie, "Operation and Control of hybrid AC/DC transmission grids," IECON 2019 - 45th Annual Conference of the IEEE Industrial Electronics Society, Lisbon, Portugal, 2019, pp. 4842-4847.

[4] E. Unamuno, J.A. Barrena, "Hybrid ac/dc microgrids - Part II: Review and classification of control strategies," Renewable and Sustainable Energy Reviews, vol. 52, December 2015, pp. 1123-1134.

[5] S. M. Malik et al, "A Generalized Droop Strategy for Interlinking Converter in a Standalone Hybrid Microgrid," Applied Energy, vol. 226, 2018, pp. 1056-1063.

[6] P. Rault, X. Guillaud, F. Colas and S. Nguefeu, "Investigation on interactions between AC and DC grids," 2013 IEEE Grenoble Conference, Grenoble, 2013, pp. 1-6.

[7] S. Martínez, F. Torres, C. Roa and E. López., "Interaction Between AC Grids and MTDC Systems Based on Droop Controllers," 2018 IEEE International Conference on Automation/XXIII Congress of the Chilean Association of Automatic Control (ICA-ACCA), Concepcion, 2018, pp. $1-6$.

[8] S.M. Malik, "Voltage and frequency control strategies of hybrid AC/DC microgrid: a review," IET Generation, Transmission \& Distribution, vol. 11, no. 2, 2017, pp. 303-313.

[9] S. Akkari, J. Dai, M. Petit and X. Guillaud, "Interaction between the voltage-droop and the frequency-droop control for multi-terminal HVDC systems," in IET Generation, Transmission \& Distribution, vol. 10, no. 6, 2016, pp. 1345-1352.

[10] P. C. Loh et al, "Autonomous Control of Interlinking Converter With Energy Storage in Hybrid AC-DC Microgrid," in IEEE Transactions on Industry Applications, vol. 49, no. 3, May-June 2013, pp. 1374-1382.

[11] P. Wang et al, "Hybrid AC/DC Micro-Grids: Solution for High Efficiency Future Power Systems," in N.R. Karki et al. (eds.), Sustainable Power Systems, Reliable and Sustainable Electric Power and Energy Systems Management, Springer, 2017.

\footnotetext{
${ }^{8}$ Note that a set is invariant if it is both positively and negatively invariant. Hence for an asymptotically stable linear system with constant input the only trajectories that are in a compact invariant set are equilibrium points.

${ }^{9}$ Note that the passivity properties used will also hold about $\tilde{x}^{*}$ due to the linearity of the dynamics considered.
}

[12] F. Luo et al, "A Hybrid AC/DC Microgrid Control Scheme With VoltageSource Inverter-Controlled Interlinking Converters", Power Electronics and Applications (EPE'16 ECCE Europe), 18th European Conference on, 2016.

[13] H. Yoo, T. Nguyen and H. Kim, "Consensus-Based Distributed Coordination Control of Hybrid AC/DC Microgrids," in IEEE Transactions on Sustainable Energy, vol. 11, no. 2, April 2020, pp. 629-639.

[14] J. Watson and I. Lestas, "Frequency and voltage control of hybrid AC/DC networks," in 57th IEEE Conference on Decision and Control, Dec. 2018.

[15] Omar Kotb, Mehrdad Ghandhari, Robert Eriksson, and Vijay K. Sood, "On small signal stability of an AC/DC power system with a hybrid MTDC network", Electric Power Systems Research, vol. 136, 2016, pp. 79-88.

[16] M. Hadjikypris, O. Marjanovic and V. Terzija, "Damping of inter-area power oscillations in hybrid AC-DC power systems based on supervisory control scheme utilizing FACTS and HVDC," 2016 Power Systems Computation Conference (PSCC), Genoa, 2016, pp. 1-7.

[17] M. A. Elizondo et al., "Interarea Oscillation Damping Control Using High-Voltage DC Transmission: A Survey, in IEEE Transactions on Power Systems, vol. 33, no. 6, Nov. 2018, pp. 6915-6923.

[18] T. Jouini et al, "Grid-Friendly Matching of Synchronous Machines by Tapping into the DC Storage," IFAC-PapersOnLine, vol. 49, issue 22, 2016, pp. 192-197.

[19] C. Arghir, T. Jouini, F. Dörfler, "Grid-forming control for power converters based on matching of synchronous machines," Automatica, vol. 95, 2018, pp. 273-282.

[20] L. Harnefors, A. G. Yepes, A. Vidal and J. Doval-Gandoy, "PassivityBased Controller Design of Grid-Connected VSCs for Prevention of Electrical Resonance Instability," in IEEE Transactions on Industrial Electronics, vol. 62, no. 2, Feb. 2015, pp. 702-710.

[21] L. Harnefors, X. Wang, A. G. Yepes and F. Blaabjerg, "Passivity-Based Stability Assessment of Grid-Connected VSCs - An Overview," in IEEE Jour. Emerging and Selected Topics in Power Elec., vol. 4, no. 1, March 2016, pp. 116-125.

[22] L. Harnefors, R. Finger, X. Wang, H. Bai and F. Blaabjerg, "VSC InputAdmittance Modeling and Analysis Above the Nyquist Frequency for Passivity-Based Stability Assessment," in IEEE Transactions on Industrial Electronics, vol. 64, no. 8, Aug. 2017, pp. 6362-6370.

[23] A. Kasis, E. Devane, C. Spanias, and I. Lestas, "Primary frequency regulation with load-side participation - Part I: stability and optimality," IEEE Trans. Power Systems, vol. 32, no. 5, Sept. 2017, pp. 3505-3518.

[24] C. Spanias and I. Lestas, "A system reference frame approach for stability analysis and control of power grids," IEEE Trans. Power Sys., vol. 34, no. 2, March 2019, pp. 1105-1115.

[25] Y. Gu, W. Li and X. He, "Passivity-Based Control of DC Microgrid for Self-Disciplined Stabilization," in IEEE Transactions on Power Systems, vol. 30, no. 5, Sept. 2015, pp. 2623-2632.

[26] R. Soloperto et al, "A Passivity-Based Approach to Voltage Stabilization in DC Microgrids," 2018 Annual American Control Conference (ACC), Milwaukee, WI, 2018, pp. 5374-5379.

[27] M. Cucuzella, K. Kosaraju, J. Scherpen, "Distributed Passivity-Based Control of DC Microgrids", 2019 American Control Conference (ACC), Philadelphia, PA, USA.

[28] J. Sun, "Small-Signal Methods for AC Distributed Power Systems-A Review," in IEEE Transactions on Power Electronics, vol. 24, no. 11, Nov. 2009, pp. 2545-2554.

[29] H. K. Khalil, Nonlinear Systems, 3rd edition, Prentice Hall, 2002.

[30] A. Riccobono and E. Santi, "A novel Passivity-Based Stability Criterion (PBSC) for switching converter DC distribution systems," 2012 TwentySeventh Annual IEEE Applied Power Electronics Conference and Exposition (APEC), Orlando, FL, 2012, pp. 2560-2567.

[31] J. Watson, Y. Ojo, C. Spanias and I. Lestas, "Stability of power networks with grid-forming converters," IEEE Powertech Milan, 2019.

[32] J. Machowski, J. Bialek, and D.J. Bumby. Power system dynamics: stability and control (2nd ed.). Wiley, 2008.

[33] P. Vorobev, et al, "High-Fidelity Model Order Reduction for Microgrids Stability Assessment," in IEEE Transactions on Power Systems, vol. 33, no. 1, Jan. 2018, pp. 874-887.

[34] J. Rocabert, A. Luna, F. Blaabjerg and P. Rodríguez, "Control of Power Converters in AC Microgrids," in IEEE Transactions on Power Electronics, vol. 27, no. 11, Nov. 2012, pp. 4734-4749.

[35] R.A. Horn and R.J. Johnson. Matrix analysis. Cambridge University Press, 2013.

[36] T.M. Haileselassie and K. Uhlen, "Impact of DC Line Voltage Drops on Power Flow of MTDC Using Droop Control," IEEE Transactions on Power Systems, vol. 27, no. 2, August 2012, pp. 1441-1449. 
[37] W. M. Haddad and V. Chellaboina, Nonlinear Dynamical Systems and Control: A Lyapunov-Based Approach. Princeton, U.K.: Princeton Univ. Press, 2008. 